University of South Carolina

Scholar Commons

6-24-1985

\title{
Effects of Dissipation and Temperature on Macroscopic Quantum Tunneling
}

S. Washburn

Richard A. Webb

University of South Carolina - Columbia, webbra@mailbox.sc.edu

R. F. Voss

S. M. Faris

Follow this and additional works at: https://scholarcommons.sc.edu/phys_facpub

Part of the Physics Commons

Publication Info

Published in Physical Review Letters, ed. Gene D. Sprouse, Volume 54, Issue 25, 1985, pages 2712-2715. Washburn, S., Webb, R. A., Voss, R. F., \& Faris, S. M. (1985). Effects of dissipation and temperature on macroscopic quantum tunneling. Physical Review Letters, 54(25), 2712-2715. DOI: 10.1103/

PhysRevLett.54.2712

(C) Physical Review Letters, 1985, American Physical Society

This Article is brought to you by the Physics and Astronomy, Department of at Scholar Commons. It has been accepted for inclusion in Faculty Publications by an authorized administrator of Scholar Commons. For more information, please contact digres@mailbox.sc.edu. 


\title{
Effects of Dissipation and Temperature on Macroscopic Quantum Tunneling
}

\author{
S. Washburn, R. A. Webb, R. F. Voss, and S. M. Faris ${ }^{(a)}$ \\ IBM T. J. Watson Research Center, Yorktown Heights, New York 10598 \\ (Received 26 December 1984)
}

\begin{abstract}
Measurements of the tunneling rate $\Gamma$ out of the zero-voltage state for several $\mathrm{Nb}$ edge junctions with differing shunt capacitances are described. At zero temperature, increasing the shunt capacitance lowers $\Gamma$ in agreement with dissipative calculations of the macroscopic-quantum-tunneling rate. As temperature increases, $\ln [\Gamma(T) / \Gamma(0)]_{\propto} T^{2}$ as recently predicted.
\end{abstract}

PACS numbers: $74.50 .+\mathrm{r}, 05.30 .-\mathrm{d}, 05.40 .+\mathrm{j}$

Quantum mechanics must hold for the large systems as well as for small ones. Most measured attributes of large systems result from incoherent averages of quantum-mechanical processes. In macroscopic systems having sufficient internal correlation, however, a single dynamical variable describes a large number of particles. ${ }^{1}$ Following the introductory theoretical work of Leggett ${ }^{1}$ and Kurkijärvi, ${ }^{2}$ several experiments ${ }^{3-6}$ have been performed to observe the quantum behavior of such a macroscopic dynamical variable. Most have concentrated on macroscopic quantum tunneling (MQT) of either the magnetic flux confined by a SQUID ring ${ }^{3}$ or the superconducting phase is an isolated Josephson junction. ${ }^{4-6}$ In the latter experiment, the junction is prepared in the metastable zero-voltage state, and the escape rate into the voltage state is measured as a function of temperature and bias current. At higher temperatures, escape proceeds by classical thermal activation (TA) over the effective barrier. This process is exponentially reduced as the temperature decreases, and below some temperature, quantum-mechanical tunneling through the barrier must dominate the transition rate. Caldeira and Leggett $^{7}$ showed that the dissipation inherent in macroscopic systems reduces the quantum escape rate below the predictions for the frictionless system. It was this theory, including dissipation, that was qualitatively vindicated by the early experiments. ${ }^{5,6}$ Using the same formalism, Chang and Chakravarty ${ }^{8}$ have recently performed numerical calculations which provide theoretical predictions for the $(T=0)$ MQT rate in the presence of arbitrary dissipation. Of more interest is the recent theoretical attention to finite-temperature corrections to MQT. Several groups ${ }^{9-12}$ have predicted that finite temperature enhances the MQT rate in the presence of dissipation. In the following we will describe experiments designed to test the above predictions.

The collective phase difference $\phi$ of superconducting pairs across a narrow barrier (a Josephson junction) is analogous to the position coordinate of a microscopic particle in the tilted periodic potential

$$
U(\phi)=-\left(I_{0} \Phi_{0} / 2 \pi\right)(x \phi+\cos \phi) .
$$

$I_{0}$ is the junction critical current, $\Phi_{0}=h / 2 e$, and
$I=x I_{0}$ is the current bias. For $0<x<1$, the potential consists of a series of wells, and for underdamped junctions, there are two allowed dynamical states. These correspond to a particle either trapped in one of the wells (the zero-voltage state) or sliding down the potential (the voltage state). The oscillation frequency of the particle about the well minimum is $\omega_{0}=\omega_{\mathrm{J}}(1$ $\left.-x^{2}\right)^{1 / 4}$, where $\omega_{\mathrm{J}}^{2}=2 \pi I_{0} / C \phi_{0}$, and $C$ is the effective parallel capacitance across the junction. Because of the available, lower-energy voltage state, the zero-voltage state is metastable. For a system prepared in the zero-voltage state, the thermal-activation rate out of the minimum is $\Gamma_{T}=A_{T} \exp \left(-U_{0} / k T\right)$. The prefactor $A_{T}$ is roughly $\omega_{0} / 2 \pi$, the rate of escape attempts, and $U_{0}$ is the height of the barrier. The effective shunt resistance $R$ leads to dissipative corrections ${ }^{13,14}$ to $A_{T}$. As $T \rightarrow 0$, escape from the zero-voltage state can only occur via quantum-mechanical tunnneling at a rate $\Gamma_{Q}=A_{Q} e^{-B}$. For a microscopic particle, $B \simeq U_{0} / \hbar \omega_{0}$, and $A_{Q} \simeq \omega_{0} / 2 \pi$. For a macroscopic system, however, the quantum-mechanical-tunneling rate is suppressed by dissipation ${ }^{7}$; both $A_{Q}$ and $B$ depend upon the dimensionless dissipation $\alpha$ $=\left(2 R C \omega_{0}\right)^{-1}$. Extensions of the theory to finite temperatures ${ }^{9-12}$ suggest that $B$ is also a function of $T$. Specifically, the temperature of the thermal bath enhances the quantum tunneling rate in the form $\ln \left[\Gamma_{Q}(T) / \Gamma_{Q}(0)\right]=s(\alpha) T^{2}$. The simple sum of a constant $\Gamma_{Q}$ and TA would, in contrast, be exponentially small at low $T$.

To test these predictions several nominally identical $\mathrm{Nb}$ edge junctions ${ }^{15}$ were fabricated as shown in Fig. 1. Each junction was 1.2 by $0.13 \mu \mathrm{m}$ with an oxide thickness of $20-25 \AA$. In order to systematically vary the dissipation $\alpha$, shunt capacitors of different sizes (oxide thickness $400 \AA$ ) were formed in parallel with the junctions. ( $C$ was chosen over $R$ as a variable to avoid parasitic inductances.) Stray capacitance plus the intrinsic capacitance of the junction was calculated from the device geometry and the critical current density ${ }^{16}$ as $C_{0}=0.02 \pm 0.005 \mathrm{pF}$. The shunt capacitances ranged from 0.025 to $12 \mathrm{pF}$. The larger dimensions and oxide thickness of the shunting capacitors allowed accurate determination (within $\leq 6 \%$ ) of $C$ from geometric measurements. At sufficiently small 


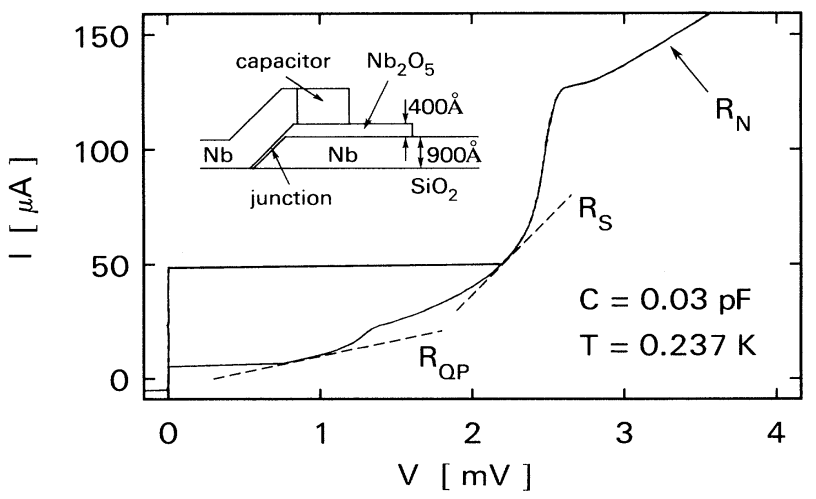

FIG. 1. Typical low-temperature $I-V$ characteristic illustrating the quasiparticle shunting resistance $R=\partial V / \partial I$. The inset is a schematic side view of the $\mathrm{Nb}$ edge junction with parallel capacitance.

plasmon frequencies $\omega_{0}$ and spatial sizes, we expect that the only effect of the shunt capacitance is to increase the total junction capacitance in the widely accepted lumped circuit [resistively shunted junction (RSJ)] model.

Figure 1 contains a typical $I-V$ curve. Almost identical characteristics were obtained for all of the junctions; the critical currents were all within $10 \%$ of 55 $\mu \mathrm{A}$, and quasiparticle resistances varied by no more than $10 \%$. The most noticeable effect of the shunt capacitors on the $I-V$ curves was in the amount of hysteresis in the return to the zero-voltage state. Slight changes in the subgap resonance structure ${ }^{17}$ were also observed with changing capacitance.

The junctions were cooled by contact with the dilute solution in a dilution refrigerator. With use of a technique described previously, ${ }^{4,5}$ measurements of the transition rate out of the zero-voltage state were performed as a function of temperature and current bias for all of the junctions. Starting in the zero-voltage state, the bias was increased slowly until the junction switched to the voltage state. The current at which it switched was recorded, and the junction was reset. This process was repeated $\sim 10^{5}$ times so that a histogram, the switching distribution $P(I)$, was obtained. Extreme care was taken to isolate the junctions from noise. Figure 2 contains distribution widths $\Delta x$ for several capacitances and representative raw data $P(I)$ for one junction. As $T$ decreased $\Delta x$ decreased, and the center of the distribution shifted toward $x=1$. As expected for MQT, ${ }^{7,8}$ below some temperature, the width and position became nearly independent of $T$, and for larger $C$, the limiting $(T=0) \Delta x$ was smaller.

The tunneling rate $\Gamma$ is obtained from the distribution by ${ }^{4}$

$$
\Gamma(x)=\frac{(d x / d t) P(x)}{1-\int_{0}^{x} P(x) d x} .
$$

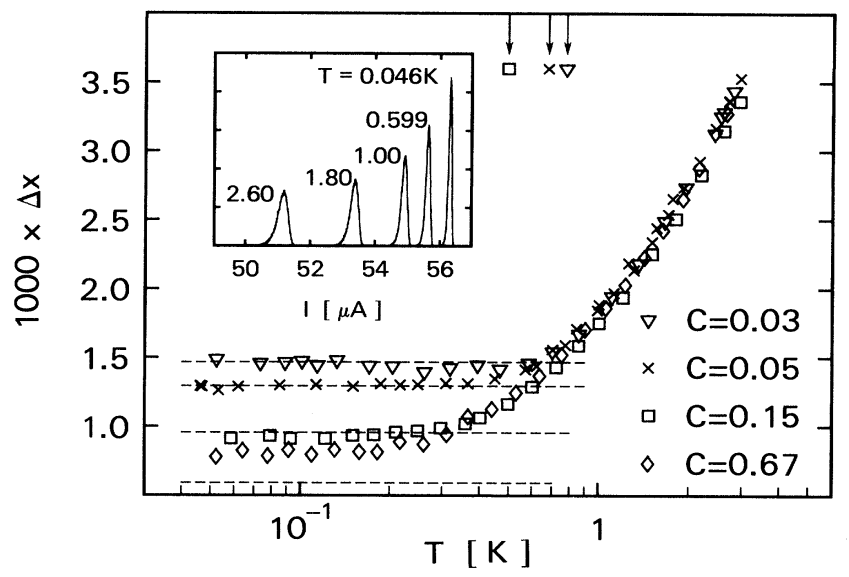

FIG. 2. The widths of the transition distributions for several junction capacitances. The various dashed lines are the theoretical predictions (Ref. 8) for MQT at $T=0$. The inset contains representative switching distributions for $C=0.15 \mathrm{pF}$. The arrows at the top are the predicted crossover temperatures for the first three junctions.

In Fig. 3, some of the experimental rates $\Gamma$ are compared with thermal-activation and MQT theories. The capacitance used in the theoretical curves was calculated from micrographs of the junctions and from material parameters used during growth of the devices. ${ }^{16}$ For high-current-density junctions, the effective capacitance is enhanced by quasiparticle tunneling. ${ }^{18}$ In our case the increase is $\delta C \simeq 0.006 \mathrm{pF}$. The resistance was measured directly from the slope of the $I-V$ characteristic above the gap $\left(R_{N}\right.$ in Fig. 1). The only remaining parameter is $I_{0}$ which is obtained from fits to thermal activation ${ }^{13,14}$ above the crossover tempera-

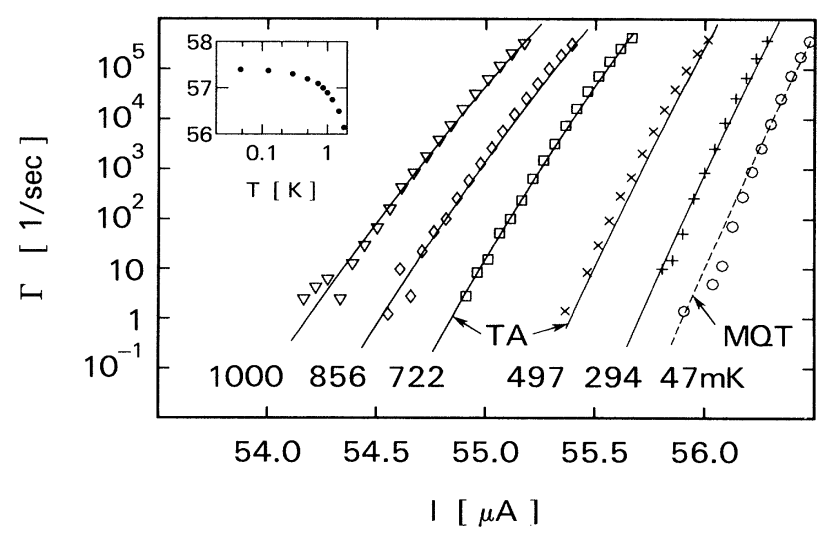

FIG. 3. Comparison of the experimental transition rate with the theory for thermal activation (solid lines) at high temperatures and for MQT (dashed line) at low temperature for $C=0.15 \mathrm{pF}$ and $R=23 \Omega$. The inset contains the fitted $I_{0}$ (in microamperes) vs $T$. 


$$
\begin{aligned}
& \text { ture }^{9-11} \\
& \qquad T_{0}=\left(\hbar \omega_{0} / 2 \pi k_{\mathrm{B}}\right)\left[\left(1+\alpha^{2}\right)^{1 / 2}-\alpha\right] .
\end{aligned}
$$

Quantum tunneling enhances the escape rate even in the regime where thermal activation dominates. (This can be seen in the experiment as a rounding of the crossover from MQT to TA in Fig. 2.) The rate enhancement may be written phenomenologically as a reduction in the barrier height ${ }^{10} U_{0} \rightarrow U_{0}-\hbar^{2} \omega_{0}^{2} /$ $12 k_{\mathrm{B}} T$. This approximation is valid far enough above $T_{0}$. For $T_{0}<T \simeq 2 T_{0}$, the true correction is slightly larger than the approximation, and is available only in numerical tables. ${ }^{9}$ The escapes rates were fitted with use of these corrections to capacitance and barrier height, and the resulting $I_{0}$ are displayed in the inset. (There is a small variation in $I_{0}$ above $T=1 \mathrm{~K}$ which remains unexplained. It may be related to the temperature renormalization of junction impedances predicted recently. ${ }^{19}$ ) Below $T \simeq T_{0}, I_{0}$ is nearly constant and agrees with the $T=0$ value. The remaining, small $(\leqslant 0.2 \%)$ variation may be an artifact of the inaccuracy of the above approximation to the quantum correction to $U_{0}$. For the three lowest-capacitance junctions, the experimental $\Gamma$ and $\Delta x$ (Fig. 2) are in excellent agreement with the magnitude and the functional form predicted by the two theories in their regions of validity. These data are the first which confirm the moderate-dissipation-regime calculations of MQT at $T=0$. The $T=0$ rate was independent of the patterned capacitance between $0.67<C<12 \mathrm{pF}$. For $C \gtrsim 0.6 \mathrm{pF}$, where the agreement with theory is not as good, calculations indicate that the lumped circuit model may break down because the patterned shunt capacitance is spatially so large that it behaves as a distributed capacitance and inductance. A new model for a junction attached to such a transmission line is necessary. 20

Theoretical work ${ }^{7-11}$ has relied heavily on the RSJ model. (Calculations that do not follow the RSJ model $^{21}$ have offered no prescription for measuring the effective $R$ from the $I-V$ curve.) Since the $I-V$ curve in Fig. 1 differs from the RSJ prediction, it is not obvious that $R_{N}$ measures the damping which appears in the theory. In fact, since the relevant dissipation is that for small oscillations about the well minimum $(V=0)$, it is surprising that $R_{N}$ (the dissipation above the gap: $V>2 \Delta / e$ ) fits the theory. We can only say that the internal consistency of the data implies that $R_{N}$ is the best measure of the damping in this experiment (as it was in the earlier experiment ${ }^{5}$ ). Not only does $R_{N}$ correctly predict the magnitude and the slope of the MQT rate, but it also correctly gives the crossover temperatures for the three junctions. For completeness, we note that theoretical fits of slightly lower quality can be obtained by using the unrenormalized capacitance and the phenomenological quasiparticle resistance $R_{S}$ measured near the switch out of the zero-voltage state (see Fig. 1). No selfconsistent choice of junction parameters would fit if $R_{\mathrm{QP}}$ (measured near the return to the zero-voltage state) was used as the resistance.

Even at very low temperatures where TA is negligible, coupling of the metastable quantum-mechanical system to a thermal bath should increase the tunneling rate ${ }^{9-12}$ as $\exp \left(T^{2}\right)$ for small $T$. According to Refs. 9 and 10, the $T^{2}$ dependence should be observed regardless of the amount of damping or the form of the potential. The only criterion is that the dissipation be Ohmic, i.e., that $R C$ approaches a constant as $\omega \rightarrow 0$, and as long as this is satisfied, the $T^{2}$ dependence of the rate is universal. ${ }^{9}$ To test the dependence of $\Gamma_{Q}$ on temperature, it is necessary to study the behavior at fixed bias $I=x I_{0}$. Figure 4 displays $\ln \Gamma(T)$ vs $T^{2}$ for the three junctions at $x=0.97$. At low $T^{2}$, the data fall on straight lines as predicted. The region of the $T^{2}$ dependence is at low temperature where the fitted $I_{0}$ is constant: The data in Fig. 4 are not an artifact of the variations in $I_{0}$. This is the first experimental observation of this behavior. The region of $T^{2}$ behavior was largest for the lowest-capacitance junction which had the highest $T_{0}$, and the region shrinks as $T_{0}$ decreases. This also is consistent with the theory. Although the $T^{2}$ dependence and its variation with $C$ are unmistakable in Fig. 4, we do not find quantitative agreement with theoretical prediction ${ }^{9}$ for the slope $s(\alpha)$. We do find, however, that $s(\alpha)$ varies approximately as predicted when $C$ changes. Although the $T^{2}$ enhancement is universal for Ohmic damping, the slope $s(\alpha)$ depends upon the form of the potential in the voltage state. That the experimental slopes are larger than the theoretical predictions is partly the result of the junction potential being larger than the approximate model

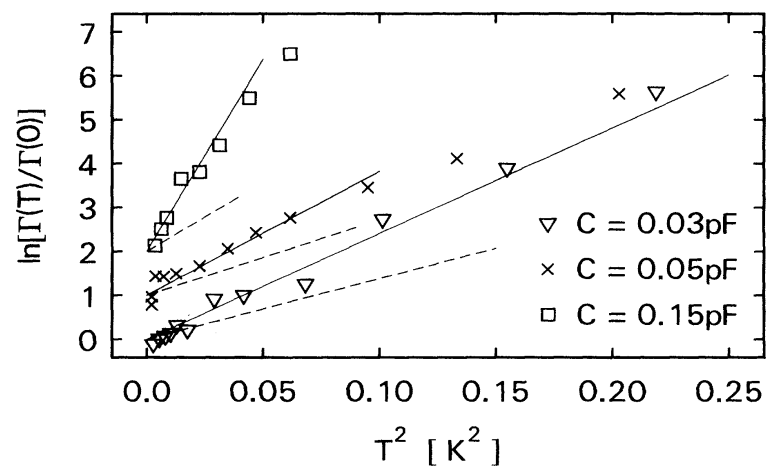

FIG. 4. The low-temperature transition rate for three junctions as a function of $T^{2}$ at $x=0.97$. The straight lines illustrate the region in temperature where the data are linear (Crosses and squares have been offset for clarity of display.) The dashed lines are the theoretical predictions for $s(\alpha)$; they are about $\frac{1}{2}$ of the experimental values. 
potential $\left[U(\phi) \sim \omega_{0}^{2} \phi^{2}-\frac{2}{3} \omega_{\mathrm{J}}^{2} x \phi^{3}\right]$ used in the calculation. Predictions for the real junction potentials would have been slightly higher. ${ }^{10}$

In summary, experiments on a series of capacitively shunted $\mathrm{Nb}$ edge junctions have qualitatively verified theoretical predictions of the $T=0$ macroscopicquantum-tunneling rate with variations in capacitance and dissipation in the range $0.2<\alpha<0.7$. This agreement was obtained with use of the renormalized capacitance and the normal-state resistance. Evidence has also been found that indicates the importance of quantum corrections to the escape process at temperatures where thermal activation dominates the escape rate. Moreover, for the first time, experimental evidence has been found to confirm the $T^{2}$ enhancement of the MQT rate from coupling to the environment.

We are grateful to M. Büttiker, A. Caldeira, S. Chakravarty, H. Grabert, P. Hanggi, A. Leggett, P. Riseborough, Y. Imry, and W. Zwerger, for instructive conversations, and to R. Drake for fabricating the junctions.

(a) Present address: Hypress, Inc., Elmsford, N.Y. 10523.

${ }^{1}$ A. J. Leggett, J. Phys. (Paris), Colloq. 39, C6-1264 (1978), and Prog. Theor. Phys., Suppl. 69, 80 (1980).

2J. Kurkijärvi, Phys. Rev. B 6, 832 (1972), and in SQUID-80: Superconducting Quantum Interference Devices and Their Applications, edited by H. D. Hahlbohm and H. Lübbig (de Gruyter, Berlin, 1980), p. 247.

$3 \mathrm{~W}$. den Boer and R. de Bruyn Ouboter, Physica (Amsterdam) 98B + C, 185 (1980); R. J. Prance et al., Nature (London) 289,543 (1981).

${ }^{4}$ T. A. Fulton and L. N. Dunkelberger, Phys. Rev. B 9 , 4760 (1974).

${ }^{5}$ R. F. Voss and R. A. Webb, Phys. Rev. Lett. 47, 265 (1981).

${ }^{6}$ L. D. Jackel et al., Phys. Rev. Lett. 47, 697 (1981).

${ }^{7}$ A. O. Caldeira and A. J. Leggett, Phys. Rev. Lett. 46, 211
(1981), and Ann. Phys. (N.Y.) 149, 374 (1983), and 153, 445(E) (1984).

${ }^{8}$ L.-D. Chang and S. Chakravarty, Phys. Rev. B 29, 130 (1984), and 30, 1566(E) (1984). For all data presented here, $\frac{1}{2} \hbar \omega_{0}<\frac{1}{3} U_{0}$, small enough to be within the range of validity of the WKB theories, which assume several states in the well.

${ }^{9} \mathrm{H}$. Grabert, U. Weiss, and P. Hanggi, Phys. Rev. Lett. 52, 2193 (1984); H. Grabert and U. Weiss, Z. Phys. B 56, 171 (1984), and Phys. Rev. Lett. 53, 1787 (1984); H. Grabert, P. Olschowski, and U. Weiss, unpublished.

10U. Weiss, P. Riseborough, P. Hanggi, and H. Grabert, Phys. Lett. 104A, 10, 492(E) (1984); P. Riseborough, P. Hanggi, and E. Friedkin, Phys. Rev. A 32, 489 (1985); P. Hanggi, H. Grabert, G.-L. Ingold, and U. Weiss, to be published.

${ }^{11}$ A. I. Larkin and Yu. N. Ovchinnikov, Pis'ma Zh. Eksp. Teor. Fiz. 37, 322 (1983) [JETP Lett. 37, 382 (1983)], and Zh. Eksp. Teor. Fiz. 85, 1510 (1983), and 86, 719 (1984)

[Sov. Phys. JETP 58, 876 (1983), and 59, 420 (1984)].

12W. Zwerger, Phys. Rev. A 31, 1745 (1985).

${ }^{13}$ M. Büttiker, E. P. Harris, and R. Landauer, Phys. Rev. B 28, 1268 (1983), and references cited therein.

${ }^{14}$ P. Hanggi and U. Weiss, Phys. Rev. A 29, 2265 (1984).

${ }^{15}$ R. F. Broom, A. Oosenbrug, and W. Walter, Appl. Phys. Lett. 37, 237 (1980).

16J. H. Magerlein, IEEE Trans. Magn. 17, 286 (1981); see also W. H. Henkels and C. J. Kircher, IEEE Trans. Magn. 13, 63 (1977).

${ }^{17}$ For example, L.-E. Hasselberg, M. T. Levinsen, and M. R. Samuelsen, J. Low Temp. Phys. 21, 567 (1975).

18 A. I. Larkin and Yu. N. Ovchinnikov, Phys. Rev. B 28, 6281 (1983).

${ }^{19} \mathrm{G}$. Brunk, H. Lübbig, and Ch. Zurbrügg, in Proceedings of the Seventeenth International Conference on Low Temperature Physics, Karlsruhe, West Germany, 1984, edited by U. Eckern et al. (North-Holland, Amsterdam, 1984), p. 219.

${ }^{20}$ This result agrees qualitatively with $\mathrm{S}$. Chakravarty and A. Schmid, unpublished.

${ }^{21}$ A. J. Leggett, Phys. Rev. B 30, 1208 (1984); U. Eckern, G. Schön, and V. Ambegaokar, Phys. Rev. B 30, 6419 (1984). 